\title{
Thalassemia in the emergency department: special considerations for a rare disease
}

\author{
Antoine N. Saliba ${ }^{1} \cdot$ Ali Atoui $^{2} \cdot$ Mohamad Labban $^{2} \cdot$ Hani Hamade $^{3} \cdot$ Rayan Bou-Fakhredin $^{4} \cdot$ Afif Mufarrij $^{3}$. \\ Ali T. Taher ${ }^{4}$
}

Received: 4 June 2020 / Accepted: 29 June 2020 / Published online: 3 July 2020

(C) Springer-Verlag GmbH Germany, part of Springer Nature 2020

\begin{abstract}
Thalassemia is characterized by a defect in the synthesis of one or more of the globin subunits of hemoglobin. This defect results in imbalance in the $\alpha / \beta$-globin chain ratio, ineffective erythropoiesis, chronic hemolytic anemia, and iron overload. With advances in diagnosis, treatment, and transfusion support, the prognosis of patients with thalassemia has improved over the past few decades. An increasing number of patients with thalassemia is living with long-term complications, including cardiomyopathy, chronic liver disease, endocrinopathy, and infections. In this paper, we review common complications that bring the patient with thalassemia to urgent or emergent medical attention. We also discuss the aspects of emergency care that are most relevant while caring for the patient with thalassemia in the emergency department.
\end{abstract}

Keywords Thalassemia $\cdot$ Emergency department $\cdot$ Iron overload $\cdot$ Resuscitation

\section{Introduction}

The thalassemias constitute a variable group of anemias characterized by a defect in the synthesis of one or more of the globin subunits of hemoglobin [1]. The hallmarks of this group of disorders include imbalance in the $\alpha / \beta$-globin chain ratio, ineffective erythropoiesis, chronic hemolytic anemia, and iron overload [2]. Thalassemic disorders were historically clustered in the Mediterranean region and the Middle East, sub-Saharan Africa, and East and Southeast Asia [3]. Modern migration patterns have altered the epidemiology of thalassemia to include Northern and Western Europe, North America, and Australia [2-5].

Ali T. Taher

ataher@aub.edu.lb

1 Division of Hematology, Department of Medicine, Mayo Clinic, Rochester, MN, USA

2 Department of Internal Medicine, American University of Beirut Medical Center, Beirut, Lebanon

3 Department of Emergency Medicine, American University of Beirut Medical Center, Beirut, Lebanon

4 Division of Hematology and Oncology, Department of Internal Medicine, American University of Beirut Medical Center, Beirut, Lebanon
The thalassemia spectrum is clinically divided into two main categories based on patients' needs for blood transfusion $[2,6]$. Patients with transfusion-dependent thalassemia (TDT) commonly present in early childhood with severe anemia that requires lifelong regular transfusion therapy for survival [2]. Patients with non-transfusion-dependent thalassemia (NTDT) present either later in childhood or in adulthood with mild or moderate anemia that only requires occasional or intermittent transfusions in certain clinical settings $[2,6]$.

Advances in understanding the pathophysiology of thalassemic disorders have contributed to the optimization of transfusion programs and the timely implementation of iron chelation therapy [7-9]. The associated improvement in prognosis has allowed patients with thalassemia to live longer. Patients have subsequently continued to experience certain complications that are not fully reversed by most of the currently available therapies [6]. Therefore, the care for the patient with thalassemia remains unique and nuanced in both the routine and urgent clinical settings. Patients may present with symptoms of heart failure, liver cirrhosis, endocrinopathy, infections, and other complications classically associated with thalassemia. Additionally, patients with thalassemia can now live longer and healthier lives and therefore engage in activities that individuals without thalassemia pursue. This results in patients with thalassemia presenting more frequently with emergent medical concerns such as traumatic injuries, 
coronary artery disease, cancer, and other diseases that were previously less commonly encountered in this patient population. In light of the evolving landscape of thalassemia care, we review common complications that bring the patient with thalassemia to urgent or emergent medical attention. We also discuss the aspects of emergency care that are most relevant in the management of the patient with thalassemia in the emergency department (ED).

\section{Initial approach}

\section{Hemodynamically stable patients}

Completing a careful history is important when a patient with thalassemia presents to the ED. On presentation, ED personnel should ask questions related to transfusion history, iron load, iron chelation regimen, and splenectomy status. Past history of surgeries (splenectomy or cholecystectomy), fractures, infections (hepatitis B, hepatitis C, or HIV), and vaccinations (meningococcal, pneumococcal, Haemophilus influenzae $b$ and influenza vaccines) should also be documented. In the patient with history of splenectomy, clinicians should consider infections with encapsulated organisms. A careful medication history is necessary and should include antiplatelet drugs, anticoagulants, hydroxyurea, insulin, levothyroxine, estrogen replacement, testosterone replacement, luspatercept, iron chelation therapy, antiviral therapy, and antiretroviral therapy. It is important to inquire about history of travel to malaria-endemic areas.

A targeted physical examination provides clues that are important for diagnosis and management. Early detection of infection foci and early recognition of sepsis based on vital signs and physical examination (encephalopathy, focal infectious findings) are essential for prompt management with appropriate antimicrobial therapy, intravenous fluids, and other supportive measures when clinically appropriate. It is essential to recognize the increased risk of agranulocytosis or neutropenia in the febrile patient receiving iron chelation therapy with deferiprone. Jaundice may reflect ongoing hemolysis. Pubertal immaturity should prompt inquiring about other endocrine complications. Skull enlargement with accentuation of the malar and frontal prominences may suggest bone marrow expansion and suboptimal transfusion schedules. Surgical scars may help confirm the surgical history and associated likely diagnoses or complications. Abdominal examination should focus on detecting hepatosplenomegaly in the absence of more emergent abdominal concerns. Hepatomegaly may suggest chronic liver disease (cirrhosis), right-sided cardiomyopathy, or extramedullary hematopoiesis. Yersinia enterocolitica infection should be considered in patients with significant iron overload, especially while on deferoxamine therapy. Although the organism usually causes self-limited gastroenteritis and mesenteric lymphadenitis, it is associated with severe systemic yersiniosis in this patient population. Mechanical compression by extramedullary hematopoietic tumors or vertebral fractures should be considered in patients presenting with pain, sensory deficits, or focal weakness [10]. A targeted neurological examination is essential for timely and accurate diagnosis. If spinal cord compression is suspected, patients should be asked about urinary retention or incontinence, fecal incontinence, and saddle anesthesia. Checking for a weak rectal sphincter tone is an important clinical tool in this setting. Rib fractures, vertebral fractures, and stress fractures should be considered in patients with pain concerns that anatomically correspond to the clinical entities. Skin examination may reveal signs of subcutaneous or intravenous injection site infections - especially in patients receiving deferoxamine therapy. The clinician should also look for signs of infection by examining indwelling central venous catheters or implanted vascular access devices.

\section{Hemodynamically unstable and trauma patients}

Patients with thalassemia presenting with hemodynamic instability need to be resuscitated and stabilized prior to further investigation. The approach does not differ from individuals without thalassemia. Obtaining vital sign measurement at the time of arrival is crucial. This includes measurements of temperature, heart rate, blood pressure, respiratory rate, and oxygen saturation. Patients presenting asystole or pulseless electrical activity (PEA) require prompt resuscitation using the advanced cardiac life support (ACLS) protocol. The ACLS protocol is based on the delivery of high-quality cardiopulmonary resuscitation (CPR) and early defibrillation. It also mandates the appropriate use of multiple elements such as anti-arrhythmic agents, vasopressors, and mechanical ventilation [11].

For patients presenting with hypotension or shock, resuscitation efforts should be initiated prior to completion of the investigation. This involves ensuring proper ventilation and tissue oxygenation, fluid resuscitation, and vasopressor administration if needed. A measurement of lactic acid level in the blood can help identify poor tissue perfusion. A state of hemodynamic shock can result from different etiologies and can therefore be classified into four different types: hypovolemic, distributive, cardiogenic, or obstructive shock. A careful history and physical examination can help determine the causative problem, which will in turn guide further treatment [12].

Patients presenting following major traumatic events are generally approached using a systematic ABCDE approach (airway, breathing, circulation, disability, exposure). This approach was first developed in the 1950s and has been gradually revised and improved through the years. It is currently delivered by the advanced trauma life support (ATLS) courses [13]. 


\section{Problem-focused management}

\section{Anemia}

Patients with TDT often require routine blood transfusions to maintain a target hemoglobin range of 9.5-10 g/dl [14]. Patients with NTDT have a broad array of stable hemoglobin levels between 6 and $9 \mathrm{~g} / \mathrm{dl}$ and often only require intermittent transfusion [14]. To maintain a steady state of hemoglobin in TDT patients, a complete blood count (CBC) should be ordered prior to each transfusion. Patients with TDT are dependent on blood transfusions with a tight schedule every 3-4 weeks and a relatively large transfusion volume of up to $20 \mathrm{~mL} / \mathrm{kg}$ [14]. Therefore, any delay in the schedule because of logistic challenges or intolerance may lead the patient to present to the ED with symptoms of anemia. Other patients may present to the ED with severe anemia secondary to delayed transfusion reactions or worsening anemia secondary to blood loss [15].

Complications associated with blood transfusion significantly contribute to morbidity from thalassemic disorders. These include delayed hemolytic transfusion reactions, alloimmunization, acute transfusion reactions, transfusiontransmitted infections, and transfusion-induced iron overload. Febrile and allergic reactions typically happen during or directly following transfusion. These include septic transfusion reactions and febrile non-hemolytic transfusion reactions. However, the presentation of immune-mediated hemolytic anemia may be acute or delayed up to 14 days. Alloimmunization is primarily caused by red blood cell (RBC) antigen incompatibility between recipient and donor $[16,17]$. Alloimmunization has been reported to be more prevalent in patients with thalassemia when compared to patients with hematologic malignancies and the general population $[18,19]$. In delayed transfusion hemolytic reactions, alloantibodies formed may lead to hyperhemolysis of both donor and host RBC [20]. Symptoms on presentation to the ED include fever, pain, and dark urine. Laboratory assessment may show a new RBC allo- or autoantibody, low hemoglobin, elevated indirect bilirubin, and hemoglobinuria [14]. Delayed transfusion hemolytic reactions and hyperhemolytic syndrome require early recognition in the ED since it is essential to avoid $\mathrm{RBC}$ transfusions as much as possible and start immunosuppressive treatment. Corticosteroids, intravenous immunoglobulin (IVIG), and rituximab have been reported to control the process of hemolysis successfully [21]. Early recognition, timely resuscitation, and appropriate involvement of the transfusion service are key steps in the ED. Other transfusion-related complications that could be encountered in the ED include transfusion-related acute lung injury and transfusion-associated circulatory overload. To mitigate the risk of transfusion-related complications, patients with TDT should usually receive blood that is at most 10 days old, negative for Kell antigen, and matched for extended rhesus phenotype.
Blood loss from gastrointestinal and other sources should be explored with thorough history and possibly other investigations when a patient with thalassemia presents to the ED with symptomatic anemia. Patients with NTDT may present to the ED with anemia secondary to aplastic crisis in the setting of parvovirus B19 infection, other infections, delayed transfusion reactions, hypersplenism, nutritional deficiency (folate), or glucose 6-phosphate dehydrogenase enzyme deficiency. RBC transfusion should be reserved for the patient with hemodynamic instability or profuse hemorrhage. It is important to establish that the patient's hemoglobin is significantly lower than their baseline, obtain an adequate transfusion history (with a history of reactions if applicable), and order a comprehensive red cell phenotype study [15]. In the setting of urgent need for blood transfusion, waiting for extended red cell antigen testing is not recommended as long as standard routine ABO typing, antibody screening, and crossmatching tests are performed. Management of the patient with thalassemia presenting to the ED with symptomatic anemia is summarized in Fig. 1.

\section{Sepsis}

Infection is one of the leading causes of mortality in patients with thalassemia [22, 23]. Factors associated with increased risk for infectious complications include splenectomy, transfusions, and iron overload [24]. Severe infections in patients with thalassemia presenting to the ED could rapidly progress to sepsis and septic shock; therefore, the clinician in the ED should be aware of the commonly implicated infectious foci and organisms. Biliary tract infection, pneumonia, soft tissue infection, and liver abscesses were the most commonly reported sites of bacterial infections [25]. Klebsiella pneumoniae, Escherichia coli, Streptococcus pneumoniae, Salmonella typhi, Yersinia enterocolitica, and other Gram-negative bacteria were the main culprits of bacterial infections [24-27]. The presentation of Yersinia enterocolitica may mimic appendicitis and can cause a severe systemic infection in patients with thalassemia.

It is crucial to consider meningitis in patients with history of splenectomy. Because of extramedullary erythropoiesis, patients with NTDT frequently have distorted sinuses and consequently develop sinusitis, which increases the risk of meningitis and brain abscess formation. A high clinical suspicion is necessary when a patient with thalassemia is presenting to the ED with headache and sinus pressure, with or without fever. When severe headache is the presenting symptom, it is important to complete a detailed history and physical examination including an evaluation for meningeal signs. A computed tomography (CT) of the head would be the next step to evaluate for intracranial bleed and stroke. If the CT is unrevealing, a lumbar puncture is recommended to evaluate for meningitis/encephalitis. A magnetic resonance imaging 


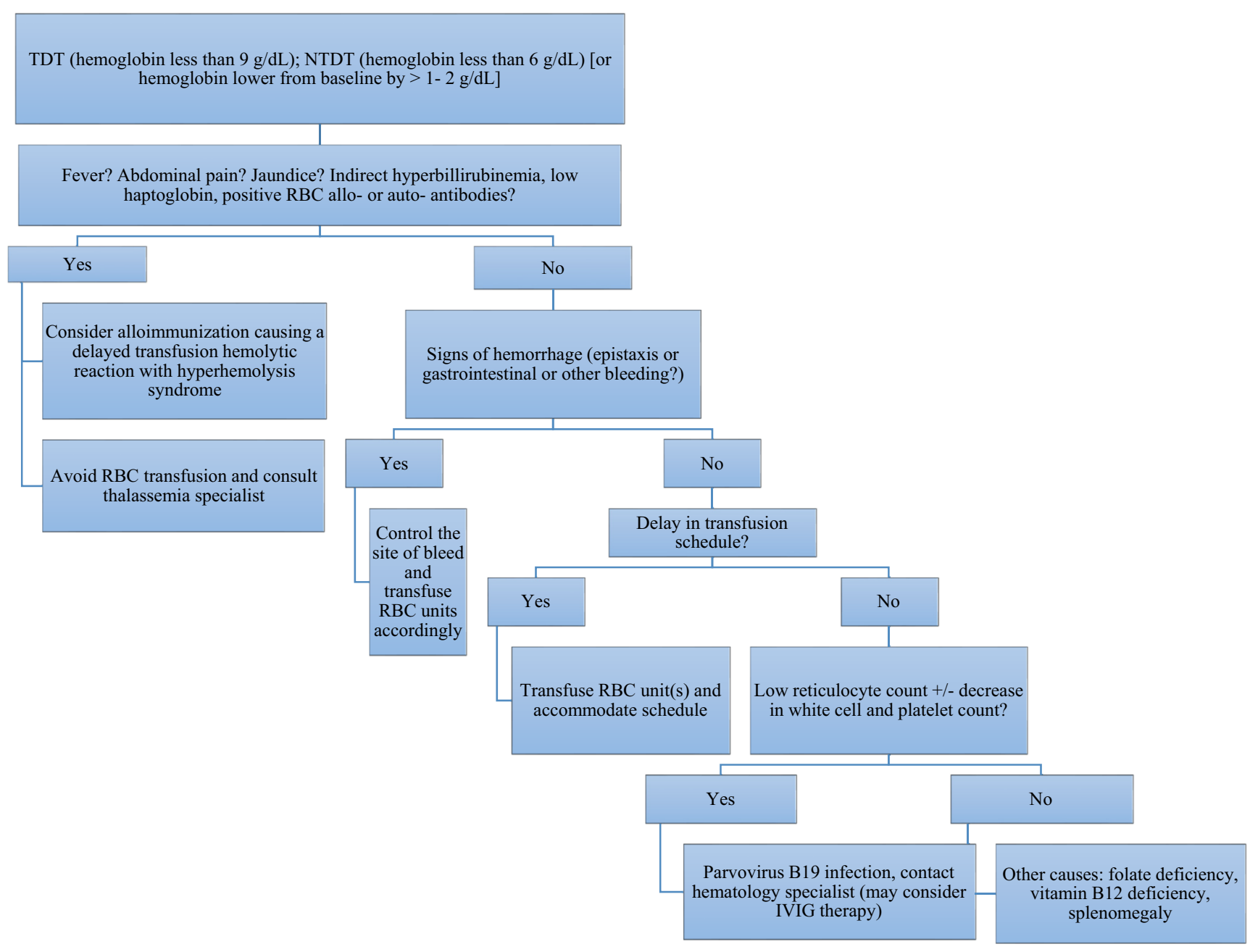

Fig. 1 Management of the patient with thalassemia presenting to the ED with symptomatic anemia. TDT transfusion-dependent thalassemia, NTDT nontransfusion-dependent thalassemia, $I V I G$ intravenous immunoglobulins

(MRI) study may be subsequently required given its higher sensitivity in evaluating for brain abscesses [15].

Recognizing sepsis and initiating intravenous fluid resuscitation and appropriate antimicrobial therapy are timesensitive priorities. Evidence strongly suggests that delays (beyond $1 \mathrm{~h}$ ) in administration of antibiotics are associated with higher mortality rate [28]. The adequacy of the antibiotic choice is also highly important, although deciding on the most appropriate agent is a complex decision. The choice of antibiotic should depend on the suspected source of infection, the common pathogens in the hospital and the community, the age and comorbidities of the patient, and any immunocompromising factors (HIV infection, neutropenia, splenectomy, etc.) [29]. Patients with suspected acute septicemia should receive broad-spectrum intravenous antibiotics covering for Gram-negative pathogens including Klebsiella and for encapsulated organisms (Streptococcus pneumoniae, Haemophilus influenzae, and Neisseria meningitides) - especially in the patient with history of splenectomy. Any sign of a systemic infection in an asplenic individual should be considered a medical emergency. The risk of postsplenectomy sepsis in patients with TDT is 30 -fold higher than in controls [30]. Fluid resuscitation, while being a priority, should be reevaluated frequently given the risk of cardiomyopathy and volume overload in the patient with thalassemia.

When suspecting a severe infection, it is important to follow a systematic approach. The patient who is well-appearing with normal vital signs and a clear infectious focus that can be treated with oral antimicrobials can be discharged from the ED with appropriate follow-up. Patients with thalassemia who present to the ED with fever need a thorough evaluation. Evaluating for common viral illnesses like seasonal influenza is important given the availability of antiviral treatment. Basic investigations include culture (urine, blood with special media for Yersinia, and stool), complete blood count with differential, chemistries, and urine analysis. Patients treated with deferiprone are at risk for agranulocytosis and neutropenic fever syndromes. If, despite basic investigations, a focus is still unclear, further investigations such as echocardiography for endocarditis, panoramic radiography for 
dental abscesses, and lumbar puncture for meningitis should be considered. After evaluating for an infection, the presentation of fever could be attributed to other conditions such as drug-related fever, thromboembolic events, and delayed transfusion reactions.

In summary, clinicians in the ED should maintain a heightened index of suspicion for the increased risk of infection in patients with thalassemia, especially in those with history of splenectomy. Therefore, it is crucial to initiate an appropriate workup and a timely plan of management for the patient with thalassemia presenting to the ED with a clinical picture suggestive of sepsis. Figure 2 summarizes the management of the patient with thalassemia presenting to the ED with suspected sepsis.

\section{Heart failure}

Despite advances in iron chelation therapy and in the therapeutic management of TDT, cardiovascular disease still remains the leading cause of morbidity and mortality in this patient population $[9,22,23,31,32]$. The onset of cardiac complications has continued to shift to older ages, with a mean age in the third decade of life [9, 31, 33, 34].

In order to address emergencies associated with heart failure in patients with TDT, understanding of the different phenotypes of heart failure encountered in these patients and their pathophysiology is necessary. In terms of ventricular function, two different phenotypes can be identified: a dilated cardiomyopathy phenotype with decreased contractility and left ventricular dilatation and a restrictive cardiomyopathy phenotype with reduced left ventricular filling and subsequent pulmonary hypertension, right ventricular dilatation, and global/ biventricular heart failure $[35,36]$. The presence of two distinct phenotypes could explain the complexity of the underlying pathophysiology. Iron deposition in the myocardium is the main cause of cardiac dysfunction. This is explained by the free plasma iron, which, in case of iron overload and complete transferrin saturation, will be present in high titers and will enter the myocytes through voltage-dependent L-type calcium channels to cause myocytes apoptosis [37]. Cardiomyopathy is more common in patients with TDT as compared to patients with NTDT. Iron overload differentially involves the liver rather than the myocardium in patients who are not dependent on regular red blood cell transfusion. This was evident from observational studies that showed absence of cardiac siderosis even in NTDT patients with severe liver iron overload [38].

Patients with heart failure in the setting of TDT present with worsening dyspnea, orthopnea, paroxysmal nocturnal dyspnea, dry cough, chest discomfort, decreased exercise tolerance, increased abdominal girth, and/or lower extremity edema. Although these symptoms may overlap with other complications of thalassemia such as venous thromboembolism and infection, clinicians in the ED should consider heart failure as an important item on the differential - even in younger patients. A basic metabolic profile (renal function markers and electrolytes - including magnesium), liver enzyme and bilirubin levels, a complete blood count, a brain natriuretic peptide (BNP) level, and an electrocardiogram should be checked. Point of care cardiac ultrasonography may help elucidate the diagnosis. Consideration should be given to checking troponin concentration. Severe anemia may aggravate heart failure symptoms and acute kidney injury. Thyroid function testing should be considered in case of tachy- or brady-arrhythmias. Timely diuresis alleviates congestive

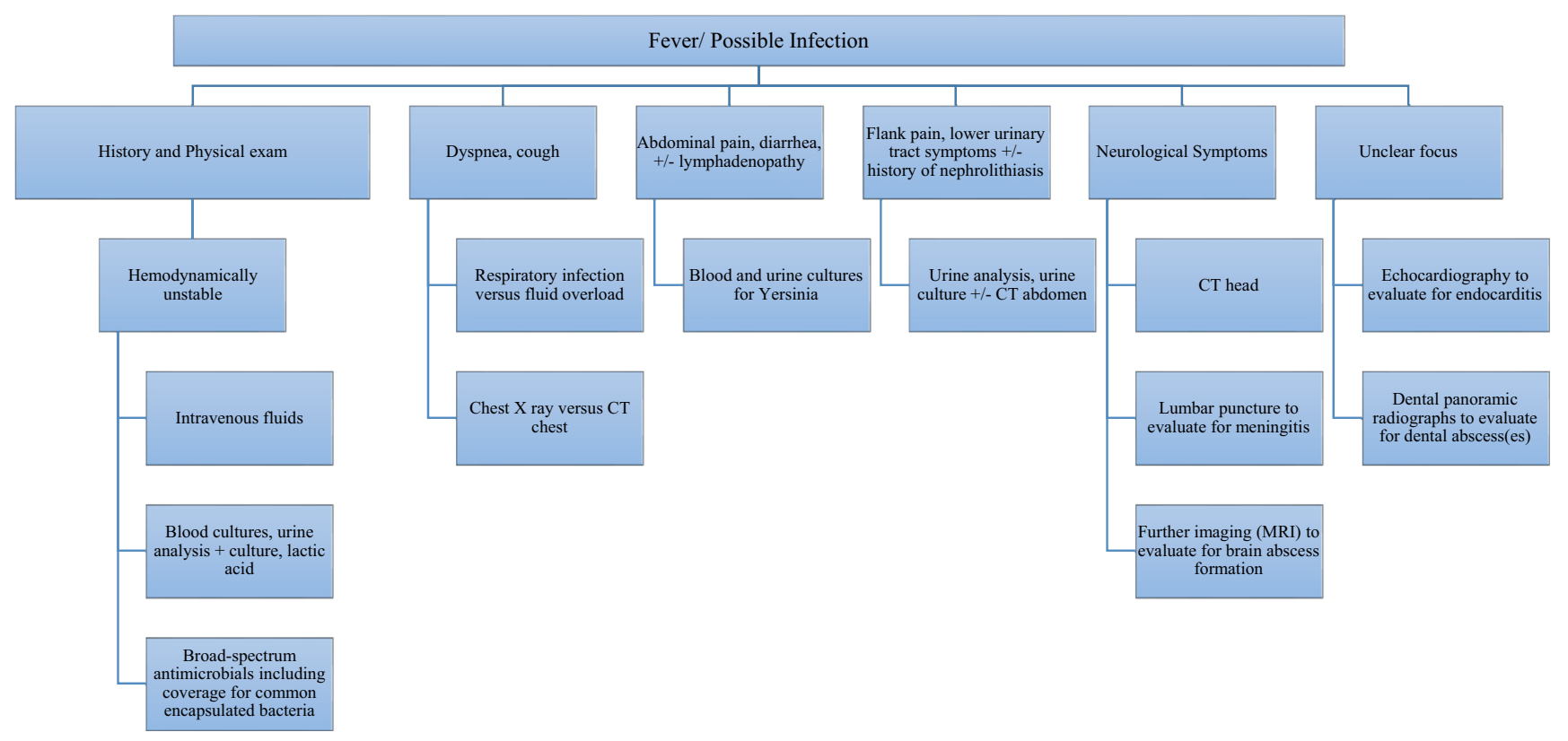

Fig. 2 Management of the patient with thalassemia presenting to the ED with suspected sepsis 
symptoms. Over-diuresis can precipitate acute renal failure. Patients with thalassemia in heart failure are sensitive to hypovolemia as a result of the restrictive physiology and stiff vasculature. In the acute setting, furosemide drips can be easier to titrate than bolus diuretics. Inotropic support should be considered in the patient presenting in cardiogenic shock. Patients with liver disease from iron overload, hepatitis C, or heart failure may have impaired hepatic synthetic function. Albumin replacement may help boost low oncotic pressures temporarily and facilitate more effective diuresis, especially in the setting of concomitant liver cirrhosis. Noninvasive positive pressure ventilation therapy may be considered in the patient with significant hypoxemia and/or hypercapnia. Admission to a unit with cardiac monitoring is recommended, while diuresis is continued.

When in the ED, patients with acute heart failure from iron overload who are off iron chelation therapy should be started on treatment. Deferoxamine should be given continuously, either subcutaneously or through a percutaneous intravenous catheter, until the ventricular function normalizes. The usual recommended dose is $40-50 \mathrm{mg} / \mathrm{kg} /$ day as a continuous intravenous infusion. However, reversibility of iron-induced cardiomyopathy after the introduction of therapy is not universally accepted due to high mortality of patients presenting with advanced heart failure despite chelation therapy [39]. In other patients, even after significant myocardial iron toxicity, aggressive iron chelation can restore myocardial function [40]. Cardiac MRI and complete echocardiography may be considered in the inpatient, outpatient, or post-acute care setting, but appropriate management in the ED should not be delayed for those investigations.

\section{Arrhythmias}

Patients with TDT are prone to arrhythmias with a mixture of triggered and reentrant types [41]. Patients with thalassemia presenting to the ED with arrhythmias are approached in a similar fashion to patients without thalassemia. Vital signs and an electrocardiogram (ECG) should be obtained, and the patient should be placed on a heart rate monitoring device. Intra-atrial reentrant tachycardia and atrial fibrillation are the most common arrhythmias detected in patients with thalassemia [18, 42]. Young, anemic patients with atrial fibrillation are more likely to be symptomatic given their relative state of hyperdynamic circulation. Atrial fibrillation needs to be managed by rate controlling agents, iron chelation in the case of iron overload, anticoagulation (after patient is screened for bleeding risk), and rhythm control agents [43]. In the case of unstable patients with supraventricular arrhythmias (patients with hypotension, chest pain, altered mental status, signs of acute heart failure), immediate cardioversion should be performed. In stable patient, pharmacological treatment can be pursued [44]. Involving a cardiologist with experience in cardiac electrophysiology is recommended. Thyroid function testing should be considered in the case of tachy- or brady-arrhythmias.

Ventricular rhythm disturbances are more specific for iron toxicity [42]. In fact, the presence of couplets, non-sustained ventricular tachycardia, or mixture of ventricular premature contractions should raise clinical suspicion for cardiomyopathy related to iron overload [42]. Amiodarone is the drug of choice for rhythm control in the acute setting because of its broad spectrum of action and relatively modest negative inotropic impact on ventricular function. In case the patient is unstable, immediate cardioversion is performed [45]. Although currently less common, heart block is another serious complication that should be rapidly recognized and managed in the ED [46].

\section{Venous and arterial thromboembolism}

Thromboembolic events are among the most serious complications in thalassemia [47]. Thromboembolic events are 4.38 times more frequent in NTDT than in TDT, with more venous thromboembolism occurring in NTDT and more arterial embolism occurring in TDT [48]. This increased incidence of thromboembolic events is thought to be related to several factors. Patients with thalassemia have high rates of platelet consumption, low levels of protein $\mathrm{C}$ and $\mathrm{S}$, and increased activation of inflammatory and endothelial cells $[49,50]$. In NTDT, high serum ferritin (> $1000 \mathrm{ng} / \mathrm{mL}$ ), age above 35 , and splenectomy are associated with a higher risk for thrombosis. Protective factors include a history of blood transfusions and hemoglobin of $9 \mathrm{~g} / \mathrm{dL}$ or greater [51]. Patients with thalassemia and, especially those with NTDT, presenting to the ED with unilateral extremity edema, warmth and/or erythema, dyspnea, chest pain, and/or hypoxia should be urgently investigated for DVT or pulmonary embolism (PE) [52].

Patients with thalassemia may present with severe headache. In this case, a physical exam should focus on looking for signs of increased intracranial pressure, such as papilledema, nausea, vomiting, and altered mental status. Clinicians in the ED should consider the possibility of central venous sinus thrombosis which could be evaluated with magnetic resonance venous imaging (MRV), usually following a computed tomography of the head (CT) to evaluate for intracranial hemorrhage. Initiation of anticoagulation therapy for venous sinus thrombosis is crucial to avoid serious neurologic complications and should be ideally discussed with the treating hematologist. Consultation with neurology and neuroradiology may help identify opportunities for intervention that may slow down or more effectively reverse the thrombotic process and its neurologic sequelae.

In addition to DVT and PE, patients with thalassemia are at higher risk of portal vein thrombosis, stroke, and silent brain ischemia $[48,53]$. Arterial thromboembolic/ischemic events, 
specifically cerebrovascular accidents, are more likely to occur in patients with TDT. Recent observational data suggest that arterial thromboembolic events are more common in patients with NTDT when compared with subjects without thalassemia [54]. Patients with thalassemia, who present to the ED with signs and symptoms of stroke, including focal somatosensory neurological deficit, should be assessed with an urgent CT of the brain to rule out intracranial bleeding. Standard stroke care should be promptly initiated including systemic thrombolytic therapy if appropriate.

\section{Trauma}

In the setting of trauma, routine resuscitation procedures should be followed. We recommend adhering to the advanced trauma life support (ATLS) guidelines and promptly involving the trauma and the general surgery teams [55]. Evaluation for worsening anemia secondary to blood loss is a key step. Whenever possible, the clinician should ensure that the hemoglobin is not lower than the steady state in the hemodynamically stable patient. Blood product and fluid support should be provided to maintain adequate organ perfusion in the hemodynamically compromised patient. Consultation with the transfusion service or the blood bank specialist is essential given the higher rates of alloimmunization in patients with thalassemia. Effective communication usually expedites the delivery of safe and appropriate blood products from the blood bank. Extended red cell phenotyping is not usually possible when emergent transfusions are needed, but blood bank specialists can usually provide safe products based on joint decision-making with the clinician. Clinical judgment should be exercised to weigh the urgency of transfusion in the setting of trauma. No patient with thalassemia should be denied a potentially lifesaving blood transfusion because of challenges in procuring compatible products. Similarly to patients without thalassemia, patients with a confirmed $\mathrm{ABO}$ type can receive uncross-matched, type-specific blood. Patients without an available ABO type can receive type $\mathrm{O}$ blood. Transfusing with the "least incompatible" blood may be necessary in patients with severe bleeding who have multiple alloantibodies, alloantibodies to high-frequency red cell antigens, or autoantibodies.

Considering splenic rupture in patients with splenomegaly is important. Given the higher prevalence of osteoporosis in patients with thalassemic disorders, clinicians should remember that bone fractures can happen with relatively minor trauma [56]. Common sites include the upper extremities, the ribs, the spine, the hips, and the metatarsals [57].

\section{Symptom-focused management}

\section{Dyspnea}

The patient living with thalassemia who presents to the ED with dyspnea should be considered at high risk for venous thromboembolism, acutely decompensated heart failure, and dysrhythmias. Patients with thalassemia, especially NTDT, are at an increased risk for venous thromboembolism and pulmonary hypertension. D-dimer assays have not been systematically studied in patients with thalassemia. Starting with a chest X-ray and an electrocardiogram is an important step.

Another rare entity that should be considered is pleural effusions related to extramedullary hematopoiesis, especially in the patient with NTDT [58]. These patients can present with hemothorax [59], and the effusions are usually exudative. Management of extramedullary hematopoiesis-related pleural effusion in the ED consists of supplemental oxygen, fluid aspiration, and blood transfusion. Antibiotic therapy should be considered if the effusion is suspected to harbor an infection or to be parapneumonic. Treatment of these pleural effusions usually requires blood transfusion to mitigate the hypoxic stimulus for extramedullary hematopoiesis, hydroxyurea therapy, radiation therapy, frequent aspiration, and/or pleurodesis $[58,59]$. Table 1 summarizes the differential diagnosis for the patient with thalassemia presenting to the ED with acute dyspnea.

\section{Chest pain}

Given the increased risk for cardiac arrhythmia, pulmonary embolism, and heart failure, patients with thalassemia who present to the ED with chest pain often warrant a comprehensive workup for life-threatening diagnoses including ventricular arrhythmias, acute coronary syndromes, pulmonary embolism, pericardial tamponade, and tension pneumothorax [42, 48, 51, 52]. Evaluation should include an electrocardiogram and a chest Xray. Despite the lack of specific risk assessment tools for venous thromboembolism in the setting of thalassemia, it is important to recognize the increased risk of venous thromboembolism in patients with thalassemia in general and NTDT in particular $[48,51]$. The clinician in the ED should examine the chest wall for areas of palpable tenderness that could be suggestive of rib fractures. Acute heart failure may present with chest discomfort or chest pain, but it may be associated with abdominal pain, especially in the epigastrium and the right upper quadrant, secondary to distension of the liver capsule in the setting of venous congestion $[22,23,33,36]$.

\section{Abdominal pain}

Patients with thalassemia are at an increased risk of cholelithiasis, portal vein thrombosis, mesenteric artery thrombosis, nephrolithiasis, and Yersinia infection [24, 25, 60]. It is important 
Table 1 Differential diagnosis for the patient with thalassemia presenting with acute dyspnea
Head, ear, nose, throat, neck

\begin{tabular}{ll}
\hline $\begin{array}{l}\text { Chest wall } \\
\text { Pulmonary }\end{array}$ & $\begin{array}{l}\text { Rib fractures } \\
\text { Bronchitis, pneumonia, pulmonary embolism, pleural effusion (parapneumonic or related to } \\
\text { extramedullary hematopoiesis), pneumothorax, asthma, chronic obstructive pulmonary } \\
\text { disease, pulmonary hypertension }\end{array}$ \\
Acute coronary syndromes, acute decompensation of chronic heart failure, cardiomyopathy, \\
high-output heart failure, flash pulmonary edema, dysrhythmia, pericarditis, cardiac \\
tamponade, myocarditis \\
Anemia
\end{tabular}

to keep these clinical entities in mind when evaluating the patient with thalassemia for abdominal pain. Figure 3 summarizes the suggested management of the patient with thalassemia presenting to the $\mathrm{ED}$ with abdominal pain.

\section{Back pain}

The patient with thalassemia presenting to the ED with acute back pain should be evaluated for the presence of neurological deficits $[10,61,62]$. The presence of focal motor or sensory deficits with or without urinary retention or bowel incontinence raises the suspicion for cord compression by extramedullary hematopoietic lesions or an acute vertebral fracture [61, 63, 64]. Presence of neurological deficits should prompt urgent consultation of a neurologist, neurosurgeon, and potentially a radiation oncologist. MRI of the spine with contrast is the imaging modality of choice to diagnose spinal cord compression [65]. Treatment includes intravenous glucocorticoids, surgical

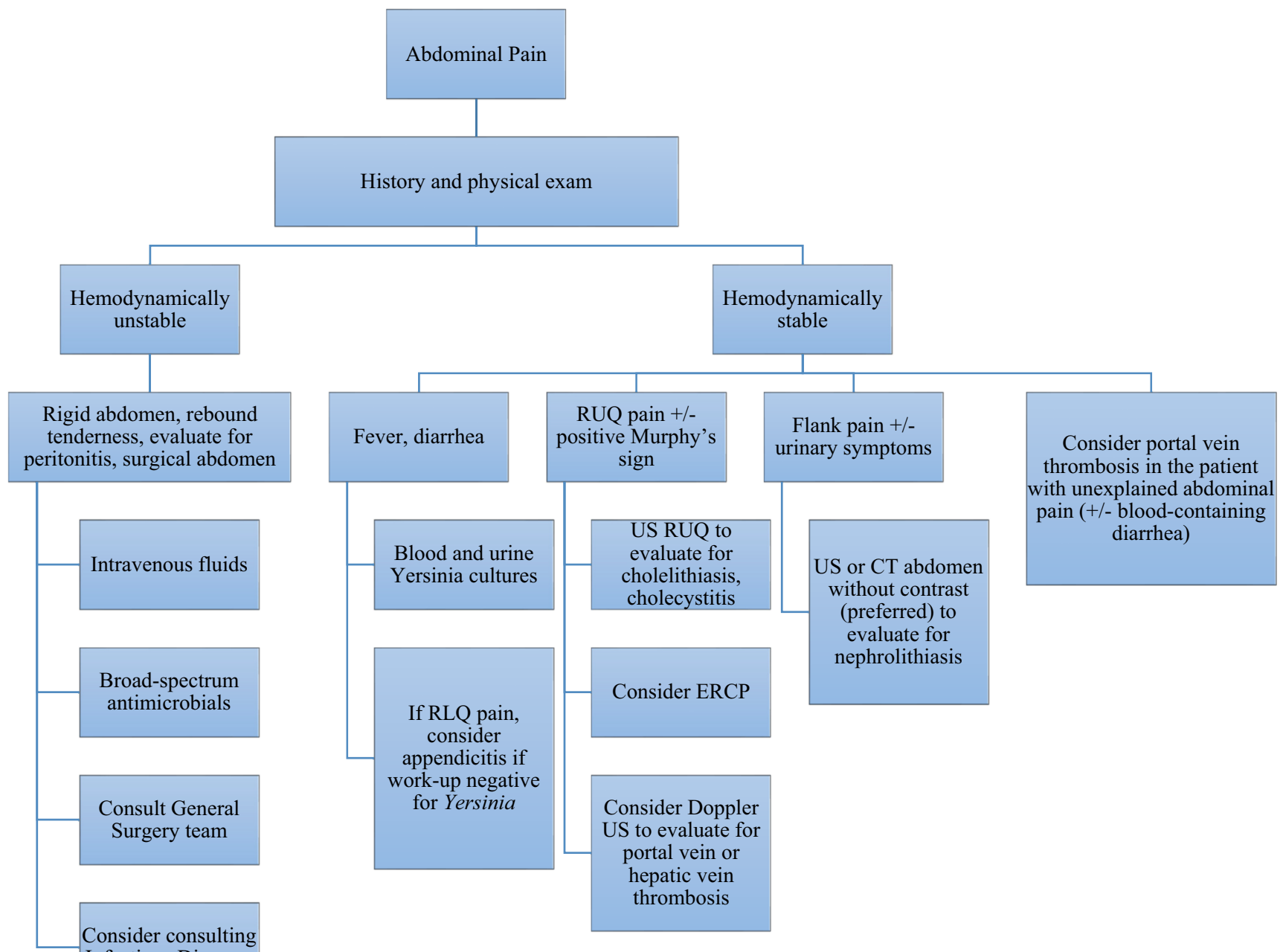

Fig. 3 Management of the patient with thalassemia presenting to the ED with abdominal pain 
decompression, radiation therapy, and blood transfusion to alleviate the hypoxic stimulus for extramedullary hematopoiesis [66, 67]. In the absence of focal neurological deficits, considering acute or chronic vertebral fractures is important as patients with thalassemia are at an increased risk of osteoporosis [68-71].

\section{Special considerations during the COVID-19 pandemic}

The current coronavirus disease 2019 (COVID-19) outbreak, caused by the novel severe acute respiratory syndrome coronavirus 2 (SARS-CoV-2), has posed novel challenges and considerations for the care of patients in the ED. Patients with thalassemia have comorbidities, including ineffective erythropoiesis, chronic hemolytic anemia, iron overload, and hypercoagulability, that may make them vulnerable to complications of SARSCoV-2 infection [72]. Although splenectomy is not known to increase the risk of severe viral infections, splenectomized patients may be at risk of severe secondary bacterial infections when infected by SARS-CoV-2. Prompt recognition of the risk of bacterial infections is important for timely institution of antibiotic therapy. A retrospective cohort of 11 Italian patients with thalassemia reported that patients with thalassemia had a generally mild clinical presentation [73]. Among this group of patients, six required hospital admission and none died [73]. Another retrospective analysis of Iranian patients with thalassemia with confirmed (15 patients) and suspected (8 patients) SARS-CoV-2 infections reported higher rates of mortality (26.1\%), when compared to experience in the Italian series and the general population in Iran [74]. Patients with NTDT were at higher risk of death compared to patients with TDT [74]. Diabetes, heart disease, and pulmonary hypertension were significantly associated with mortality [74]. Given the limited clinical experience with SARSCoV-2 in patients with thalassemia, general recommendations for the general population must be followed with special attention to thrombotic and secondary bacterial infectious complications. Vigilance in instituting early isolation of patients with thalassemia from any potentially infected contacts remains important. Avoiding nonurgent transfusion support in the ED is important to keep patients with thalassemia away from high-risk settings. Triaging patients and determining the safest disposition should follow general guidelines for the general population. Clinicians should consider home with self-isolation for the duration of symptoms as the safest disposition for patients who are mildly symptomatic, hemodynamically stable, and without evidence of acutely exacerbated hemolysis. Given the heightened risk for acute worsening of chronic hemolysis, clinicians should consider checking blood counts for the patients with thalassemia diagnosed with SARS-CoV-2 infection. For patients with TDT, specific plans for continuation of transfusion support during the period of self-isolation should be made in consultation with the treating hematologist and the transfusion facility. There is no current evidence that COVID-19 may be transmitted through blood transfusion [75]. Care should be exercised in considering thrombotic complications in patients presenting with SARSCoV-2 infection as both thalassemia and COVID-19 have been associated with hypercoagulable states [52, 76, 77].

\section{Conclusion}

Patients with thalassemia may present to the ED with various concerns that may be unique to the disease or similar to the general population. The initial approach to the patient with thalassemia presenting to the ED should include thorough history and a targeted physical examination. Awareness of ED providers about the different complications of thalassemia may help initiate a collaborative and multidisciplinary effort with the goal of early recognition and expedited management.

\section{Compliance with ethical standards}

Conflict of interest The authors declare that they have no conflict of interest.

Ethics approval This manuscript is an expert review and is exempt of ethics approval.

\section{References}

1. Marengo-Rowe AJ (2007) The thalassemias and related disorders. Proc (Bayl Univ Med Cent) 20:27-31

2. Taher AT, Weatherall DJ, Cappellini MD (2018) Thalassaemia. Lancet 391:155-167

3. Weatherall DJ (2018) The evolving spectrum of the epidemiology of thalassemia. Hematol Oncol Clin North Am 32:165-175

4. Weatherall DJ (2010) The inherited diseases of hemoglobin are an emerging global health burden. Blood 115:4331-4336

5. Modell B, Darlison M (2008) Global epidemiology of haemoglobin disorders and derived service indicators. Bull World Health Organ 86:480-487

6. Taher AT, Cappellini MD (2018) How I manage medical complications of beta-thalassemia in adults. Blood 132:1781-1791

7. Vitrano A, Calvaruso G, Lai E, Colletta G, Quota A, Gerardi C, Concetta Rigoli L, Pitrolo L, Cuccia L, Gagliardotto F, Filosa A, Caruso V, Argento C, Campisi S, Rizzo M, Prossomariti L, Fidone C, Fustaneo M, di Maggio R, Maggio A (2017) The era of comparable life expectancy between thalassaemia major and intermedia: is it time to revisit the major-intermedia dichotomy? $\mathrm{Br} \mathrm{J}$ Haematol 176:124-130

8. Ladis V, Chouliaras G, Berdousi H et al (2005) Longitudinal study of survival and causes of death in patients with thalassemia major in Greece. Ann N Y Acad Sci 1054:445-450

9. Borgna-Pignatti C, Rugolotto S, De Stefano P et al (2004) Survival and complications in patients with thalassemia major treated with transfusion and deferoxamine. Haematologica 89:1187-1193

10. Nemtsas P, Arnaoutoglou M, Perifanis V, Koutsouraki E, Orologas A (2015) Neurological complications of beta-thalassemia. Ann Hematol 94:1261-1265 
11. Panchal AR, Berg KM, Hirsch KG, Kudenchuk PJ, del Rios M, Cabañas JG, Link MS, Kurz MC, Chan PS, Morley PT, Hazinski MF, Donnino MW (2019) 2019 American Heart Association focused update on advanced cardiovascular life support: use of advanced airways, vasopressors, and extracorporeal cardiopulmonary resuscitation during cardiac arrest: an update to the American Heart Association guidelines for cardiopulmonary resuscitation and emergency cardiovascular care. Circulation 140:e881-e894

12. Vincent JL, De Backer D (2014) Circulatory shock. N Engl J Med 370:583

13. Thim T, Krarup NH, Grove EL, Rohde CV, Løfgren B (2012) Initial assessment and treatment with the airway, breathing, circulation, disability, exposure (ABCDE) approach. Int J Gen Med 5: $117-121$

14. Tubman VN, Fung EB, Vogiatzi M, Thompson AA, Rogers ZR, Neufeld EJ, Kwiatkowski JL, Thalassemia Clinical Research Network (2015) Guidelines for the standard monitoring of patients with thalassemia: report of the thalassemia longitudinal cohort. J Pediatr Hematol Oncol 37:e162-e169

15. Porter J, Taher A, Mufarrij A, Gavalas M (2012) In: Eleftheriou A, Angastiniotis M, Telfer P (eds) Emergency management of thalassaemia. Thalassaemia International Federation (c) 2012 Thalassaemia International Federation, Nicosia

16. Chou ST, Liem RI, Thompson AA (2012) Challenges of alloimmunization in patients with haemoglobinopathies. Br J Haematol 159:394-404

17. Lal A, Wong TE, Andrews J, Balasa VV, Chung JH, Forester CM, Ikeda AK, Keel SB, Pagano MB, Puthenveetil G, Shah SJ, Yu JC, Vichinsky EP (2018) Transfusion practices and complications in thalassemia. Transfusion 58:2826-2835

18. Heddle NM, Soutar RL, O'Hoski PL, Singer J, McBride JA, Ali MAM, Kelton JG (1995) A prospective study to determine the frequency and clinical significance of alloimmunization post-transfusion. Br J Haematol 91:1000-1005

19. Schonewille H, Haak HL, van Zijl AM (1999) Alloimmunization after blood transfusion in patients with hematologic and oncologic diseases. Transfusion 39:763-771

20. Eberly LA, Osman D, Collins NP (2015) Hyperhemolysis syndrome without underlying hematologic disease. Case Rep Hematol 2015:180526

21. Azarkeivan A, Ansari S, Ahmadi MH, Hajibeigy B, Maghsudlu M, Nasizadeh S, Shaigan M, Toolabi A, Salahmand M (2011) Blood transfusion and alloimmunization in patients with thalassemia: multicenter study. Pediatr Hematol Oncol 28:479-485

22. Borgna-Pignatti C, Rugolotto S, De Stefano P et al (1998) Survival and disease complications in thalassemia major. Ann N Y Acad Sci 850:227-231

23. Zurlo MG, De Stefano P, Borgna-Pignatti C et al (1989) Survival and causes of death in thalassaemia major. Lancet 2:27-30

24. Rahav G, Volach V, Shapiro M, Rund D, Rachmilewitz EA, Goldfarb A (2006) Severe infections in thalassaemic patients: prevalence and predisposing factors. Br J Haematol 133:667-674

25. Wang SC, Lin KH, Chern JP et al (2003) Severe bacterial infection in transfusion-dependent patients with thalassemia major. Clin Infect Dis 37:984-988

26. Issaragrisil S, Wanachiwanawin W, Bhuripanyo K, Benjasuratwong Y, Piankijagum A, Wasi P (1987) Infection in thalassemia: a retrospective study of 1,018 patients with beta-thalassemia/Hb E disease. Birth Defects Orig Artic Ser 23:505-511

27. Wanachiwanawin W (2000) Infections in E-beta thalassemia. J Pediatr Hematol Oncol 22:581-587

28. Kumar A, Roberts D, Wood KE, Light B, Parrillo JE, Sharma S, Suppes R, Feinstein D, Zanotti S, Taiberg L, Gurka D, Kumar A, Cheang M (2006) Duration of hypotension before initiation of effective antimicrobial therapy is the critical determinant of survival in human septic shock. Crit Care Med 34:1589-1596
29. Rhodes A, Evans LE, Alhazzani W, Levy MM, Antonelli M, Ferrer R, Kumar A, Sevransky JE, Sprung CL, Nunnally ME, Rochwerg B, Rubenfeld GD, Angus DC, Annane D, Beale RJ, Bellinghan GJ, Bernard GR, Chiche JD, Coopersmith C, de Backer DP, French CJ, Fujishima S, Gerlach H, Hidalgo JL, Hollenberg SM, Jones AE, Karnad DR, Kleinpell RM, Koh Y, Lisboa TC, Machado FR, Marini JJ, Marshall JC, Mazuski JE, McIntyre LA, McLean AS, Mehta S, Moreno RP, Myburgh J, Navalesi P, Nishida O, Osborn TM, Perner A, Plunkett CM, Ranieri M, Schorr CA, Seckel MA, Seymour CW, Shieh L, Shukri KA, Simpson SQ, Singer M, Thompson BT, Townsend SR, van der Poll T, Vincent JL, Wiersinga WJ, Zimmerman JL, Dellinger RP (2017) Surviving Sepsis campaign: international guidelines for management of sepsis and septic shock: 2016. Intensive Care Med 43:304-377

30. Singer DB (1973) Postsplenectomy sepsis. Perspect Pediatr Pathol $1: 285-311$

31. Chouliaras G, Yiannoutsos CT, Berdoukas V, Ladis V (2009) Cardiac related death in thalassaemia major: time trend and risk factors in a large Greek unit. Eur J Haematol 82:381-387

32. Koohi F, Kazemi T, Miri-Moghaddam E (2019) Cardiac complications and iron overload in beta thalassemia major patients-a systematic review and meta-analysis. Ann Hematol 98:1323-1331

33. Aessopos A, Farmakis D, Hatziliami A, Fragodimitri C, Karabatsos F, Joussef J, Mitilineou E, Diamanti-Kandaraki E, Meletis J, Karagiorga M (2004) Cardiac status in well-treated patients with thalassemia major. Eur J Haematol 73:359-366

34. Engle MA, Erlandson M, Smith CH (1964) Late cardiac complications of chronic, severe, refractory anemia with hemochromatosis. Circulation 30:698-705

35. Spirito P, Lupi G, Melevendi C, Vecchio C (1990) Restrictive diastolic abnormalities identified by Doppler echocardiography in patients with thalassemia major. Circulation 82:88-94

36. Kremastinos DT, Tsetsos GA, Tsiapras DP, Karavolias GK, Ladis VA, Kattamis CA (2001) Heart failure in beta thalassemia: a 5-year follow-up study. Am J Med 111:349-354

37. Wood JC (2008) Cardiac iron across different transfusiondependent diseases. Blood Rev 22(Suppl 2):S14-S21

38. Taher AT, Musallam KM, Wood JC, Cappellini MD (2010) Magnetic resonance evaluation of hepatic and myocardial iron deposition in transfusion-independent thalassemia intermedia compared to regularly transfused thalassemia major patients. Am J Hematol 85:288-290

39. Cohen AR, Galanello R, Pennell DJ, Cunningham MJ, Vichinsky E (2004) Thalassemia. Hematology Am Soc Hematol Educ Program 2004:14-34

40. Wood JC, Tyszka JM, Carson S, Nelson MD, Coates TD (2004) Myocardial iron loading in transfusion-dependent thalassemia and sickle cell disease. Blood 103:1934-1936

41. Lekawanvijit S, Chattipakorn N (2009) Iron overload thalassemic cardiomyopathy: iron status assessment and mechanisms of mechanical and electrical disturbance due to iron toxicity. Can J Cardiol 25:213-218

42. Kaye SB, Owen M (1978) Cardiac arrhythmias in thalassaemia major: evaluation of chelation treatment using ambulatory monitoring. Br Med J 1:342

43. Barbero U, Fornari F, Guarguagli S, Gaglioti CM, Longo F, Doronzo B, Anselmino M, Piga A (2018) Atrial fibrillation in beta-thalassemia major patients: diagnosis, management and therapeutic options. Hemoglobin 42:189-193

44. Neumar RW, Otto CW, Link MS, Kronick SL, Shuster M, Callaway CW, Kudenchuk PJ, Ornato JP, McNally B, Silvers SM, Passman RS, White RD, Hess EP, Tang W, Davis D, Sinz E, Morrison LJ (2010) Part 8: adult advanced cardiovascular life support: 2010 American Heart Association guidelines for cardiopulmonary resuscitation and emergency cardiovascular care. Circulation 122:S729-S767 
45. Tang PT, Do DH, Li A, Boyle NG (2018) Team management of the ventricular tachycardia patient. Arrhythm Electrophysiol Rev 7:238-246

46. Maleki AR, Nikyar B, Hosseini SM (2012) Third-degree heart block in thalassemia major: a case report. Iran J Pediatr 22:260-264

47. Michaeli J, Mittelman M, Grisaru D, Rachmilewitz EA (1992) Thromboembolic complications in beta thalassemia major. Acta Haematol 87:71-74

48. Taher A, Isma'eel H, Mehio G et al (2006) Prevalence of thromboembolic events among 8,860 patients with thalassaemia major and intermedia in the Mediterranean area and Iran. Thromb Haemost 96:488-491

49. Eldor A, Durst R, Hy-Am E, Goldfarb A, Gillis S, Rachmilewitz EA, Abramov A, Maclouf J, Godefray YC, de Raucourt E, Guillin MC (1999) A chronic hypercoagulable state in patients with betathalassaemia major is already present in childhood. Br J Haematol 107:739-746

50. Sumiyoshi A, Thakerngpol K, Sonakul D (1992) Pulmonary microthromboemboli in thalassemic cases. Southeast Asian J Trop Med Public Health 23(Suppl 2):29-31

51. Taher AT, Musallam KM, Karimi M, el-Beshlawy A, Belhoul K, Daar S, Saned MSE, el-Chafic AH, Fasulo MR, Cappellini MD (2010) Overview on practices in thalassemia intermedia management aiming for lowering complication rates across a region of endemicity: the OPTIMAL CARE study. Blood 115:1886-1892

52. Cappellini MD, Motta I, Musallam KM, Taher AT (2010) Redefining thalassemia as a hypercoagulable state. Ann N Y Acad Sci 1202:231-236

53. Karimi M, Haghpanah S, Pishdad P, Zahedi Z, Parand S, Safaei S (2019) Frequency of silent brain lesions and aspirin protection evaluation over 3 years follow-up in beta thalassemia patients. Ann Hematol 98:2267-2271

54. Liu X, Wen J, Xu Y, Ruan Y, Yi T, Chen JC, Wu X (2019) Increased risk of arterial thromboembolic events in transfusionnaive thalassemia: a nationwide population-based study. J Investig Med 67:826-832

55. Galvagno SM Jr, Nahmias JT, Young DA (2019) Advanced trauma life support((R)) update 2019: management and applications for adults and special populations. Anesthesiol Clin 37:13-32

56. Voskaridou E, Terpos E (2004) New insights into the pathophysiology and management of osteoporosis in patients with beta thalassaemia. Br J Haematol 127:127-139

57. Fung EB, Harmatz PR, Milet M, Coates TD, Thompson AA, Ranalli M, Mignaca R, Scher C, Giardina P, Robertson S, Neumayr L, Vichinsky EP, Multi-Center Iron Overload Study Group (2008) Fracture prevalence and relationship to endocrinopathy in iron overloaded patients with sickle cell disease and thalassemia. Bone 43:162-168

58. Aessopos A, Tassiopoulos S, Farmakis D, Moyssakis I, Kati M, Polonifi K, Tsironi M (2006) Extramedullary hematopoiesis-related pleural effusion: the case of beta-thalassemia. Ann Thorac Surg 81: 2037-2043

59. Chu KA, Hsu CW, Lin MH, Lin SJ, Huang YL (2015) Recurrent spontaneous massive hemothorax from intrathoracic extramedullary hematopoiesis resulting in respiratory failure. $\mathrm{J}$ Formos Med Assoc 114:282-284

60. Adamkiewicz TV, Berkovitch M, Krishnan C, Polsinelli C, Kermack D, Olivieri NF (1998) Infection due to Yersinia enterocolitica in a series of patients with beta-thalassemia: incidence and predisposing factors. Clin Infect Dis 27:1362-1366

61. Taher A, Isma'eel H, Cappellini MD (2006) Thalassemia intermedia: revisited. Blood Cells Mol Dis 37:12-20

62. Nemtsas P, Arnaoutoglou M, Perifanis V, Koutsouraki E, Spanos G, Arnaoutoglou N, Chalkia P, Pantelidou D, Orologas A (2018)
Polyneuropathy and myopathy in beta-thalassemia major patients. Ann Hematol 97:899-904

63. Prabhakar S, Chopra JS, Khosla VK, Dash S, Banerjee AK (1980) Spinal cord compression in homozygous beta thalassaemia. Surg Neurol 13:351-354

64. Ricchi P, Meloni A, Grigoratos C, Toia P, Fina P, Pistoia L, Costantini S, Borsellino Z, Lisi R, Rocca M, Preziosi P, Midiri M, Pepe A (2019) Prevalence of extramedullary hematopoiesis, renal cysts, splenic and hepatic lesions, and vertebral hemangiomas among thalassemic patients: a retrospective study from the myocardial Iron overload in thalassemia (MIOT) network. Ann Hematol 98:1333-1339

65. Coskun E, Keskin A, Suzer T et al (1998) Spinal cord compression secondary to extramedullary hematopoiesis in thalassemia intermedia. Eur Spine J 7:501-504

66. Ismail II, Massoud F, Alexander KJ, Al-Hashel JY (2019) Compressive dorsal myelopathy secondary to extramedullary hematopoiesis in a thalassemic patient. Case Rep Neurol Med 2019: 5827626

67. Moncef B, Hafedh J (2008) Management of spinal cord compression caused by extramedullary hematopoiesis in beta-thalassemia. Intern Med 47:1125-1128

68. Haidar R, Mhaidli H, Musallam KM, Taher AT (2012) The spine in beta-thalassemia syndromes. Spine (Phila Pa 1976) 37:334-339

69. Haidar R, Musallam KM, Taher AT (2011) Bone disease and skeletal complications in patients with beta thalassemia major. Bone 48: 425-432

70. Noureldine MHA, Taher AT, Haydar AA, Berjawi A, Khamashta MA, Uthman I (2018) Rheumatological complications of beta-thalassaemia: an overview. Rheumatology (Oxford) 57:19-27

71. Otrock ZK, Azar ST, Shamseddeen WA, Habr D, Inati A, Koussa S, Mahfouz RAR, Taher AT (2006) Intravenous zoledronic acid treatment in thalassemia-induced osteoporosis: results of a phase II clinical trial. Ann Hematol 85:605-609

72. Taher AT, Bou-Fakhredin R, Kreidieh F, Motta I, de Franceschi L, Cappellini MD (2020) Care of patients with hemoglobin disorders during the COVID-19 pandemic: an overview of recommendations. Am J Hematol

73. Motta I, Migone De Amicis M, Pinto VM et al (2020) SARS-CoV2 infection in beta thalassemia: Preliminary data from the Italian experience. Am J Hematol

74. Karimi M, Haghpanah S, Azarkeivan A, Zahedi Z, Zarei T, Akhavan Tavakoli M, Bazrafshan A, Shirkavand A, de Sanctis V (2020) Prevalence and mortality due to outbreak of novel coronavirus disease (COVID-19) in $\beta$-Thalassemias: the nationwide Iranian experience. Br J Haematol

75. Roy NBA, Telfer P, Eleftheriou P, de la Fuente J, Drasar E, Shah F, Roberts D, Atoyebi W, Trompeter S, Layton DM, Lugthart S, Stuart-Smith S, Chakravorty S, Wright J, Porter J, Inusa B, Howard J, National Haemoglobinopathy Panel (2020) Protecting vulnerable patients with inherited anaemias from unnecessary death during the COVID-19 pandemic. Br J Haematol 189:635-639

76. Connors JM, Levy JH (2020) COVID-19 and its implications for thrombosis and anticoagulation. Blood 135:2033-2040

77. Tan CW, Low JGH, Wong WH, Chua YY, Goh SL, Ng HJ (2020) Critically ill COVID-19 infected patients exhibit increased clot waveform analysis parameters consistent with hypercoagulability. Am J Hematol 95:E156-e158

Publisher's note Springer Nature remains neutral with regard to jurisdictional claims in published maps and institutional affiliations. 